\title{
PIPERADE: A Penning-trap isobar separator for the DESIR low- energy facility of SPIRAL2
}

\author{
P. Ascher ${ }^{1, a}$, B. Blank ${ }^{2}$, K. Blaum ${ }^{1}$, P. Dupré ${ }^{3}$, M. Gerbaux², S. Grévy², H. Guérin², M. Heck \\ D. Lunney ${ }^{3}$, S. Naimi ${ }^{1}$, and A. de Roubin ${ }^{1,2}$ \\ ${ }^{1}$ Max-Planck-Institut für Kernphysik, Saupfercheckweg 1, 69117 Heidelberg, Germany \\ ${ }^{2}$ CENBG-IN2P3-CNRS, Université Bordeaux 1, 33175 Gradignan, France \\ ${ }^{3}$ CSNSM-IN2P3-CNRS, Université Paris-Sud, 91405 Orsay, France
}

\begin{abstract}
A Penning-trap isobar separator is currently under construction at CENBG (Bordeaux, France) and MPIK (Heidelberg, Germany) for a future installation at the SPIRAL2/DESIR facility. This device aims at purifying the radioactive ion beams from undesired species, in order to deliver highly pure samples of exotic nuclei to the different set-ups which will be installed in the DESIR hall. The present manuscript describes the context and the motivations to build such a system, the targeted characteristics, and the studies to find an efficient purification method for large samples of isobaric species.
\end{abstract}

\section{The DESIR facility at SPIRAL2}

The last decades have seen an extremely rapid development of Radioactive Ion Beam (RIB) facilities allowing important progress concerning the knowledge of the atomic nucleus. Exploring new territory of nuclei with extreme $N / Z$ ratios allowed the discovery of new structures and behaviors of the nucleus, thus refining the theoretical nuclear models originally developed from observations on long-lived nuclei [1]. The future SPIRAL2 facility [2] at GANIL in Caen, France, will push the limits further and allow experiments with nuclei which are currently inaccessible. The DESIR hall will be the installation dedicated to low-energy studies and will host experimental set-ups for decay spectroscopy, laser spectroscopy and trap-based experiments, in order to study nuclear ground and excited state properties. Thanks to these three complementary techniques, key observables of nuclei far from stability will be accessible, allowing to answer many of the open questions concerning the nuclear forces, the processes of nucleosynthesis as well as testing the Standard Model.

The DESIR facility will be equipped with different beam lines which will receive low-energy beams (10-60 keV) from: (i) the existing SPIRAL1 facility, (ii) the SPIRAL2 production building using neutron- and deuteron-induced fission, deep-inelastic and fusion evaporation reactions and (iii) the super separator spectrometer $S^{3}$ where neutron-deficient and superheavy elements will be produced via in-flight fusion evaporation reactions. The combination of the complementary in-flight and ISOL methods and of many different reaction mechanisms using unprecedented high intensities will make the DESIR facility a powerful and versatile low-energy facility. The construction of the DESIR hall will begin in 2016 .

\footnotetext{
ae-mail: pauline.ascher@mpi-hd.mpg.de
} 


\section{The PIPERADE set-up}

The PIPERADE set-up (PIège de PEnning pour les RAdioisotopes à DEsir, i.e. Penning trap for radioactive ions at DESIR) [3] will be a high-resolution separator and accumulator placed upstream the DESIR hall.

\subsection{Motivations}

Most of the experiments within the DESIR program require ultra pure beams of radioactive ions. For example, this is crucial for the super-allowed $0^{+} \rightarrow 0^{+}$decay studies to reach the precision needed on the half-lives and branching-ratios for a test of the Standard Model [4]. The contamination of the radioactive beam arises mainly from non-selective ionization. For many cases the contaminants are less exotic than the ions of interest, leading to a large ratio of contamination to the desired species reaching several orders of magnitude. This makes the purification task a critical issue. In addition, in the case of the ISOL production method, chemical reactions also occur in the target producing molecular contaminations, often with high intensity.

The isotopic contaminants can easily be removed by standard magnetic dipole separators but a more powerful device is needed for close isobaric species or even isomers. Penning traps [5] are widely used as isobaric separators with a resolution of up to $10^{5}$. However, this performance is limited by the amount of stored ions in the trap, which can not exceed a thousand at most $[6,7]$. Therefore, the PIPERADE system under construction aims for purifying very large samples of ions $\left(>10^{4}\right)$ while maintaining the resolving power necessary for isobar selection of $10^{5}$.

\subsection{Experimental set-up}

The injection into the Penning trap requires a bunched and cooled beam. For this purpose, a gas-filled Radio Frequency Quadrupole (called GPIB for General Purpose Ion Buncher) will be installed in front of the Penning trap, in order to deliver bunches with a longitudinal emittance of about $10 \mathrm{eV}$. $\mu \mathrm{s}$ and a transversal emittance reduced to $1-3 \pi \mathrm{mm}$ mrad at $60 \mathrm{keV}$ kinetic beam energy. This device is under construction at CENBG (see Figure 1, left), the design is very similar to the one of ISCOOL successfully used at ISOLDE [8]. The double-Penning trap device has been designed at MPIK (see Figure 1, right) and consists of a purification trap and an accumulation trap. With such a system, one can repeat the purification cycle by sending each time a newly cleaned sample to the second trap. Once the accumulated sample is large enough for a measurement, a final cleaning is needed to get rid of the daughter nuclei produced during the accumulation time and the high-purified sample of ions of interest can be sent to the various experiments.

In addition, the PIPERADE set-up will include an ion source to test the complete set-up at CENBG, and then to be used at DESIR to perform off-line measurements and to provide stable beams to the set-ups installed in the hall. This FEBIAD source is already in operation at CENBG and emittance measurements are ongoing to characterize the beam which will be sent to the GPIB.

\subsection{Isobar separation in a Penning trap}

The most common ion separation method using a Penning trap (like at ISOLTRAP [9] or JYFLTRAP [10]) is the sideband buffer-gas cooling technique [11]. The first step of this method is to apply a dipolar field at the frequency of the ion magnetron motion $\omega_{-}$, which is in first order mass-independent. This excitation increases the radius of all stored ions. The second step consists of applying a quadrupolar field at the true cyclotron frequency $\omega_{c}$ of the desired ions, allowing to convert the magnetron 
motion into the modified cyclotron motion. By combining this conversion with the effect of buffer gas, which cools the modified cyclotron motion by collisions, one can re-center selectively the ions of interest. By ejecting the ions through a diaphragm, only the desired species are transferred to the second trap. Nevertheless, this method is strongly limited by the number of ions [6, 7] and when the density of the trapped ion cloud increases, space charge effects get stronger, leading to frequency shifts, peak broadening and screening effects which makes the selective re-centering inefficient.
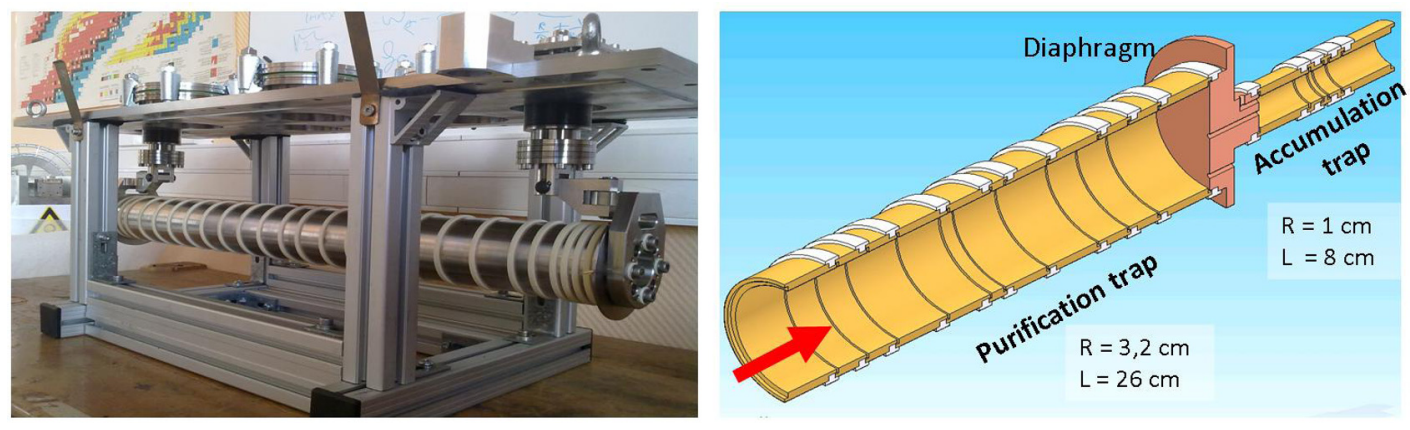

Figure 1. Left: Picture of the GPIB under construction at CENBG. Right: Drawing of the Penning-trap system under development at MPIK consisting of two traps, one for the purification and the other one for the accumulation of the ions of interest. There will be a diaphragm between both traps to act as a pumping barrier and also to transfer the ions of interest centered in the first trap.

First simulations have been performed with the SIMBUCA program [12], which directly calculates the Coulomb interaction between the particles and therefore is well suited for the investigation of space charge effects. In these simulations, an ion ensemble with $10 \%$ of ${ }^{136} \mathrm{Sb}$ and $90 \%$ of ${ }^{136} \mathrm{Te}$ are first excited for $4 \mathrm{~ms}$ at the magnetron frequency. Then a quadrupolar excitation is applied for $300 \mathrm{~ms}$ at $\omega_{c}$ of ${ }^{136} \mathrm{Sb}$, using helium as a buffer gas at a pressure of $10^{-4}$ mbar. Figure 2 shows the efficiency of the selective re-centering as a function of the total number of ions. This efficiency corresponds to the percentage of ions of interest which have, after the separation process, a motional radius smaller than $2 \mathrm{~mm}$, i.e. the ions which will be ejected from the trap through the diaphragm. Two different data sets are shown in Figure 2 corresponding to two initial spatial distributions of the ions; the black points correspond to simulations with an initial size of the cloud of $R_{\text {cloud }}=0.7 \mathrm{~mm}$ and $Z_{\text {cloud }}=0.5 \mathrm{~mm}$ whereas the red points correspond to an initial cloud size of $R_{\text {cloud }}=2 \mathrm{~mm}$ and $Z_{\text {cloud }}=15 \mathrm{~mm}$. Expanding the cloud to such an elongated cylindrical shape assumes a good homogeneity of the magnetic field $(<10 \mathrm{ppm})$ over this trapping region. The general behavior of these curves shows a sudden drop of the efficiency at a certain number of ions due to frequency shifts and screening effects. One can see that this steep decrease occurs at a lower number of ions for a more compressed cloud, thus for a higher ion density. As seen in Figure 1, the size of the purification trap will be large, in order to limit the space charge effects. By increasing the trap size, the field anhamornicities are much further from the center thus the cloud can be more easily expanded. New excitation schemes to decrease the cloud density are also under investigation. One example is the anti-rotating wall excitation technique [13], a circularly polarized field which can expand radially and axially the cloud in the trap. In addition, new separation methods like the SIMCO excitation [14, 15] are also under study at MPIK in an FT-ICR test Penning trap set-up [16], to find the most efficient and the fastest method to separate large samples of isobaric species in a Penning trap. 


\section{EPJ Web of Conferences}

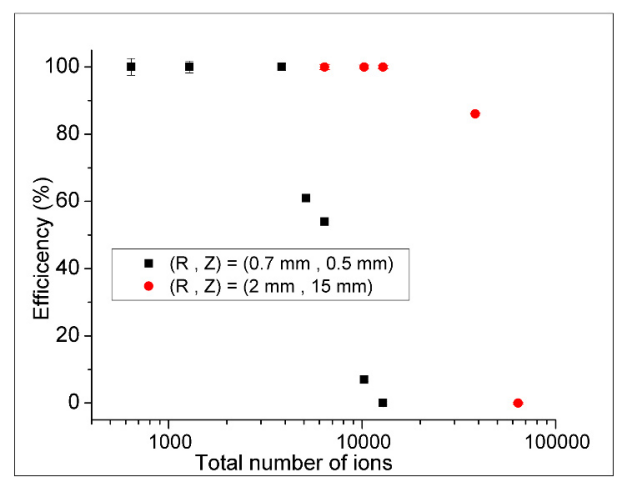

Figure 2. Efficiency of the re-centering of the ions of interest, here ${ }^{136} \mathrm{Sb}$ among $90 \%$ of contaminants $\left({ }^{136} \mathrm{Te}\right)$, as a function of the total number of ions for two initial distributions of the cloud. This efficiency corresponds to the percentage of the ${ }^{136} \mathrm{Sb}$ ions which have, after the selective centering, a motional radius smaller than $2 \mathrm{~mm}$.

\section{Conclusion}

A double Penning-trap system is being developed for the future SPIRAL2/DESIR facility. Test measurements are underway at MPIK to investigate different separation methods and simulations are in progress to study the space charge effects occuring in a Penning trap filled with large ion ensembles $\left(>10^{4}\right)$. The trap system will be built and first tested at MPIK and then moved to CENBG Bordeaux (2014-2015) where the offline ion source and the GPIB are developed for a complete test of the PIPERADE set-up before the installation at DESIR in 2017-2018.

This work was supported by the Région Aquitaine, the Agence Nationale de Recherche (ANR-2011BS04-020-02) and the Max Planck Society. P.A. acknowledges support by the Humboldt fundation. We thank the ISOLDE technical group for providing the drawings of ISCOOL.

\section{References}

[1] iks32.fys.kuleuven.be/aris2011

[2] http://www.ganil-spiral2.eu/spiral2

[3] http://www.cenbg.in2p3.fr/piperade

[4] J.C. Hardy and I.S. Towner, Phys. Rev. C 79, 055502 (2009)

[5] K. Blaum et al., Phys. Rep. 425, 1-78 (2006)

[6] A. Herlert et al., Hyp. Int. 199, 211-220 (2011)

[7] S. Van Gorp, PhD Thesis, KU Leuven (2012)

[8] H. Frånberg et al., Nucl. Instr. Meth. B 266, 4502 (2008)

[9] M. Mukherjee et al., Eur. Phys. J. A 35, 1-29 (2008)

[10] S. Rinta-Antila et al., Eur. Phys. J. A 31, 1-7 (2007)

[11] G. Savard et al., Phys. Lett. A 158, 247-252 (1991)

[12] S. Van Gorp et al., Nucl. Instr. Meth. A 638, 192200 (2011)

[13] X.-P. Huang et al., Phys. Rev. Lett. 78, 875-878 (1997)

[14] M. Rosenbusch et al., Int. J. M. Spec. 325-327, 51-57 (2012)

[15] M. Kretzschmar, Int. J. M. Spec. 325-327, 30-44 (2012)

[16] M. Ubieto Diaz et al., Int. J.M. Spec. 288, 1-5 (2009) 